\title{
Erratum: Three-Particle Correlation from a Many-Body Perspective: Trions in a Carbon Nanotube [Phys. Rev. Lett. 116, 196804 (2016)]
}

\author{
Thorsten Deilmann®, Matthias Drüppel, and Michael Rohlfing
}

( ) (Received 22 November 2019; published 20 December 2019)

DOI: 10.1103/PhysRevLett.123.259902

In a recent work, Torche and Bester [1] investigated trions by using a method which we had discussed in [2]. The authors of Ref. [1] emphasize that their method differs from our method by two additional terms (last two lines in Eq. (4) in Ref. [1]), which should be included in the Hamiltonian, but did not occur in Eq. (2) of our publication [2]. We have checked our previous work and realized that the two terms in question had already been included in our numerical treatment [2], in contradiction to Eq. (2) where they were omitted. Therefore, Eq. (2) has to be changed to

$$
\begin{aligned}
\left\langle\mathbf{v c}_{1} \mathbf{c}_{2}\left|\hat{H}^{(e e h)}\right| \mathbf{v}^{\prime} \mathbf{c}_{1}^{\prime} \mathbf{c}_{2}^{\prime}\right\rangle= & \left(\epsilon_{\mathbf{c}_{1}}+\epsilon_{\mathbf{c}_{2}}-\epsilon_{\mathbf{v}}\right) \delta_{\mathbf{c}_{1}, \mathbf{c}_{1}^{\prime}} \delta_{\mathbf{c}_{2}, \mathbf{c}_{2}{ }^{\prime}} \delta_{\mathbf{v}, \mathbf{v}^{\prime}}+\left(W_{\mathbf{c}_{1} \mathbf{c}_{2}, \mathbf{c}_{1}^{\prime} \mathbf{c}_{2}^{\prime}}-W_{\mathbf{c}_{1} \mathbf{c}_{2}, \mathbf{c}_{2}^{\prime} \mathbf{c}_{1}^{\prime}}\right) \delta_{\mathbf{v}, \mathbf{v}^{\prime}}-\left(W_{\mathbf{v}^{\prime} \mathbf{c}_{1}, \mathbf{v}_{1}^{\prime}}-V_{\mathbf{v}^{\prime} \mathbf{c}_{1}, \mathbf{c}_{1}^{\prime} \mathbf{v}}\right) \delta_{\mathbf{c}_{2}, \mathbf{c}_{2}{ }^{\prime}} \\
& -\left(W_{\mathbf{v}^{\prime} \mathbf{c}_{2}, \mathbf{v} \mathbf{c}_{2}^{\prime}}-V_{\mathbf{v}^{\prime} \mathbf{c}_{2}, \mathbf{c}_{2}^{\prime} \mathbf{v}}\right) \delta_{\mathbf{c}_{1}, \mathbf{c}_{1}{ }^{\prime}}+\left(W_{\mathbf{v}^{\prime} \mathbf{c}_{1}, \mathbf{v} \mathbf{c}_{2}{ }^{\prime}}-V_{\mathbf{v}^{\prime} \mathbf{c}_{1}, \mathbf{c}_{2}{ }^{\prime} \mathbf{v}}\right) \delta_{\mathbf{c}_{2}, \mathbf{c}_{1}}+\left(W_{\mathbf{v}^{\prime} \mathbf{c}_{2}, \mathbf{v} \mathbf{v}_{1}{ }^{\prime}}-V_{\mathbf{v}^{\prime} \mathbf{c}_{2}, \mathbf{c}_{1} \mathbf{v}_{\mathbf{v}}}\right) \delta_{\mathbf{c}_{1}, \mathbf{c}_{2}{ }^{\prime}}
\end{aligned}
$$

as well as the corresponding Eqs. (S17) and (S18) in the Supplemental Material of the original Letter [2]. Since the two terms were in fact used in our numerical treatment, all numerical results and all interpretation and conclusions of Ref. [2] (and our further works citing this reference) remain unchanged.

We thank Torche and Bester for bringing this issue to our attention.

[1] A. Torche and G. Bester, First-principles many-body theory for charged and neutral excitations: Trion fine structure splitting in transition metal dichalcogenides, Phys. Rev. B 100, 201403(R) (2019).

[2] T. Deilmann, M. Drüppel, and M. Rohlfing, Three-Particle Correlation from a Many-Body Perspective: Trions in a Carbon Nanotube, Phys. Rev. Lett. 116, 196804 (2016). 